\title{
Estudio cinético del proceso de digestión anaerobia de las aguas residuales de la elaboración de aceitunas de mesa negras
}

\author{
Por R. Borja Padilla, A. Garrido Fernández y M.M. Durán Barrantes \\ Instituto de la Grasa y sus Derivados (C.S.I.C.). Avda. Padre García Tejero, 4. 41012 - SEVILLA
}

\section{RESUMEN}

Estudio cinético del proceso de digestión anaerobia de las aguas residuales de la elaboración de aceitunas de mesa negras.

Se ha efectuado un estudio cinético del proceso de digestión anaerobia de las aguas residuales obtenidas en la elaboración de aceitunas negras, mediante biorreactores que contienen en suspensión soportes micronizados de tipo arcilloso de distinta composición química (Sepiolita y Bentonita).

Admitiendo que globalmente el proceso de digestión anaerobia sigue una cinética de primer orden, se ajustan las parejas de datos experimentales, volumen de metano acumulado $(G)$. tiempo (t) a la ecuación: $G=G_{m} \cdot\left[1-\exp \left(-K_{0} \cdot t\right)\right]$, determinándose los valores del coeficiente de velocidad $K_{a}$, para cada caso estudiado. El soporte utilizado ejerce una influencia acusada sobre la constante cinética del proceso, presentando valores significativamente mayores en el digestor con Sepiolita. Los valores medios obtenidos, dentro del intervalo de DQO comprendido entre 0 y $1 \mathrm{~g} / \mathrm{l}$ fueron: 1,83 dias " (Sepiolita) y 1,23 dias $^{1}$ (Bentonita). Para concentraciones de sustrato superiores, la constante cinética del proceso disminuye al aumentar el volumen de agua residual añadido a los digestores.

Los coeficientes de rendimiento de producción de metano, $Y_{\text {wS }}$, fue ron de 333 y $316 \mathrm{~mL} \mathrm{CH}_{4} / \mathrm{g}$ DQO para los reactores con soportes Sepiolita y Bentonita, respectivamente. Los porcentajes de eliminación de DQO son superiores al $95 \%$ en todos los casos.

PALABRAS-CLAVE: Aceituna negra de mesa - Agua residual - Cinética - Depuración anaerobia - Soporte.

\section{SUMMARY}

Kinetic study of anaerobic digestion process of wastewaters from ripe olive processing.

A kinetic study of the anaerobic digestion process of wastewaters from black olive preparation was carried out. The bioreactors used accommodated various suspended micronized clay supports of differen chemical composition (Sepiolite and Bentonite).

Assuming that the overall anaerobic digestion process conforms to a first-order kinetics, experimental data pairs, namely the methane volume yielded $(G)$ and the time (t) fitted the equation: $G=G_{m} \cdot\left[1-\exp \left(-K_{0} \cdot t\right)\right]$, the rate coefficient, $K_{0}$, was determined in each of the situations studied. The support used has a marked influence on the kinetic constant of the pro cess: the Sepiolite support yields significantly the highest values. The average $K_{0}$ values, obtained in the rank of COD between 0 and $1 \mathrm{~g} / \mathrm{L}$ were: 1.83 days $^{-1}$ (Sepiolite) and 1.23 days " (Bentonite).

For values of COD greater than $1 \mathrm{~g} / \mathrm{L}$, the specific rate constant decreased when the volume of wastewater added or substrate concentration was increased.

The yield coefficients of methane, $\mathrm{Y}_{\mathrm{\alpha}, \mathrm{s}}$, were 333 and $316 \mathrm{~mL} \mathrm{CH} / \mathrm{g}$ COD for the Sepiolite and Bentonite reactor, respectively. Treatment yields of COD were above $95 \%$ in all the cases.

KEY-WORDS: Anaerobic digestion - Kinetics - Support - Table black olive - Wastewater

\section{INTRODUCCION}

La elaboración de aceitunas de mesa se efectúa según diversos métodos. Los tres tipos más difundidos son verdes, negras y negras al natural en salmuera.

De ellos, la elaboración del tipo negras es la más extendida en USA y ha empezado a tener una amplia expansión también en España en los últimos diez años (COI, 1989). Para su preparación (Fernández Díez et al., 1985), las aceitunas se conservan en salmuera durante un período más o menos prolongado (de unos dos meses a un año), sometiéndose después a una serie sucesiva de tratamientos con disoluciones de hidróxido sódico (entre tres y cinco), seguidas de sus correspondientes lavados para eliminar el exceso de $\mathrm{NaOH}$. La duración conjunta de cada serie suele ser de unas 24 horas y durante la permanencia en agua, e incluso algunas veces durante la inmersión en lejía, se suele pasar aire con objeto de oxidar los frutos, que poco a poco van adquiriendo una tonalidad progresivamente más oscura. Al cabo de un período entre tres y cinco días, cuando el producto ha adquirido el suficiente color negro, las aceitunas se sumergen en una disolución de gluconato ferroso al $0,1 \%$ durante 24 horas, después se introducen en una salmuera de equilibrio y finalmente se envasan y se esterilizan.

Como puede desprenderse del proceso de elaboración, el volumen de aguas residuales que se producen en el transcurso del mismo es elevada, mayor que en cualquier otro tipo de aceitunas de mesa, pudiendo llegar a alcanzarse del orden de 30 litros por kilogramo de frutos.

La característica más destacada de las mismas es: la presencia de elevadas concentraciones de $\mathrm{Na}^{+}$en las salmueras y lejías, lo que hace que el contenido de este elemento en las aguas de lavado sea igualmente alto, aunque inferior al de las dos disoluciones mencionadas anteriormente. Asimismo, el conjunto de todas ellas presentan un $\mathrm{pH}$ alcalino, una presencia elevada de polifenoles polimerizados, un color oscuro indeseable y una concentración alta de hierro en las disoluciones de gluconato ferroso desechadas. Además, a diferencia de lo que ocurre con otras industrias oleícolas, su vertido se produce regularmente a lo largo de todo el año. Finalmente, su 
carga orgánica (DBO) oscila entre 2 g y $5 \mathrm{~g}\left(\mathrm{O}_{2} / \mathrm{L}\right)$ (Fernández Díez et al., 1985). Por todo ello, se han encontrado hasta ahora grandes inconvenientes a que se vierta a los cauces públicos y a que sea incorporada a las redes de saneamiento públicas. En USA la solución más usual para deshacerse de las mismas consiste en depositarlas en balsas de evaporación. La misma idea se está aplicando en España y otros países oleícolas (Israel, por ejemplo).

Además de ello, se han estudiado algunas medidas de control interno para disminuir el volumen de vertidos. De ellas, la más utilizada es el reuso de las lejías alcalinas, así como la neutralización de las aguas de lavado con ácidos clorhídrico o carbónico y la incorporación del gluconato ferroso directamente en el envasado (Fernandez Díez et al., 1985). De esta manera se ha logrado controlar la extensión de las balsas, que iba aumentando de manera alarmante a medida que se incrementaba la demanda.

En cualquier caso, la utilización de este sistema de balsas está encontrando progresiva oposición por parte de la sociedad (por los malos olores que a veces desprende, crecimiento de insectos, etc.) y de las autoridades, que ven en ellas graves riesgos de contaminación de los acuíferos subterráneos.

En consecuencia, es necesario continuar investigando el tratamiento de estos efluentes para encontrar alguna otra solución que pueda ser más aceptable. En este sentido la digestión anaerobia es un sistema que ha dado buenos resultados con una gran variedad de aguas residuales (Olthof and Oleszkiewick, 1982) (Fiestas, 1984) mostrándose eficaz en diversas condiciones de trabajo (Jeris, 1983) y cargas orgánicas (Rozzi, 1986). Su aplicación a este tipo de aguas residuales resulta, pues, atractiva. Por tanto el objetivo de este trabajo es la aplicación de la digestión anaerobia, con utilización de dos tipos diferentes de soportes, al tratamiento de las aguas residuales de la etapa de ennegrecimiento durante la fabricación de aceitunas tipo negras, estudiando los parámetros característicos del proceso y la influencia que ejercen sobre los mismos las condiciones de operación.

\section{MATERIAL Y METODOS}

\section{a) Equipo de digestión anaerobia}

Consistió en dos unidades de digestión anaerobia agitadas magnéticamente, de 1 litro de capacidad sumergidas en un baño de agua a $35^{\circ} \mathrm{C}$. Otras características y detalles de este equipo están recogidas en un trabajo precedente (Borja et al., 1990).

Para llevar a cabo dicho proceso, los microorganis. mos responsables se inmovilizan en el interior de los reactores utilizando Sepiolita y Bentonita, dos silicatos de tamaño micronizado que permiten la fijación de las bacterias metanogénicas, con objeto de evitar su pérdida en los efluentes del proceso y con ello una posible disminución de la velocidad del mismo.

\section{b) Procedimiento experimental}

La metodología utilizada para el arranque y puesta a punto de los digestores anaerobios está descrita en un trabajo precedente. Tras un período de aclimatación de dos meses, se realizan una serie de experimentos en régimen discontinuo, añadiendo volúmenes de 50,100, $150,200,250,300,350,400,450,500,550$ y $600 \mathrm{~mL}$ del agua residual objeto de estudio (Borja et al., 1990).

En todos ellos, las cargas se añaden después de retirar el mismo volumen de líquido del biorreactor, tras decantar durante dos horas para evitar pérdidas de biomasa.

En cada ensayo se mide el volumen de metano producido diariamente, así como las DQO inicial y final. La duración del mismo se corresponde con el tiempo necesario para la biometanización completa de cada carga. Todas las experiencias se realizan por duplicado.

Los analisis de los parámetros de caracterización de los distintos efluentes residuales se efectúan de acuerdo con "Standard Methods for the Examination of Water and Wastewater" (American Public Health Association, 1985).

c) Caracteristicas de las aguas residuales de la elaboración de aceitunas negras

Los valores de los principales parámetros para medir la carga contaminante de las mismas se resumen en la tabla I.

\section{RESULTADOS EXPERIMENTALES}

En las tablas $\mid 1$ y 111 se recoge el volumen de metano acumulado (mililitros, medidos a $25^{\circ} \mathrm{C}$ de temperatura media y presión atmosférica) en función del tiempo (dias), para las distintas cargas de alimentación utilizadas, en los reactores con soporte Sepiolita y Bentonita.

En la tabla IV aparecen los valores de la DQO $(\mathrm{g} / \mathrm{L})$ al inicio de cada experiencia en todos los casos estudiados.

Tabla 1

Caracteristicas del agua residual utilizada

\begin{tabular}{lc}
$\mathrm{pH}$ & 9,1 \\
Demanda Quimica de Oxígeno (DQO) & $2,50 \mathrm{~g} / \mathrm{L}$ \\
Sólidos totales & $19,80 \mathrm{~g} / \mathrm{L}$ \\
Sólidos minerales & $17,45 \mathrm{~g} / \mathrm{L}$ \\
Sólidos volátiles & $2,35 \mathrm{~g} / \mathrm{L}$ \\
Sólidos en suspensión totales & $2,20 \mathrm{~g} / \mathrm{L}$ \\
Sólidos en suspensión minerales & $1,60 \mathrm{~g} / \mathrm{L}$ \\
Sólidos en suspensión volátiles & $0,60 \mathrm{~g} / \mathrm{L}$ \\
Acidez volátil (ácido acético) & $0,56 \mathrm{~g} / \mathrm{L}$ \\
Alcalinidad (CaCO $\mathrm{CO}_{3}$ ) & $0,63 \mathrm{~g} / \mathrm{L}$ \\
Fenoles totales (ácido cafeico) & $0,30 \mathrm{~g} / \mathrm{L}$ \\
\hline
\end{tabular}


Tabla II

Volumen de metano acumulado $(\mathrm{mL})$ en función del tiempo (días).

Reactor Sepiolita

\begin{tabular}{rrrrrrrrrrr}
\hline $\begin{array}{c}\text { Volumen de agua } \\
\text { residual } \\
(\mathrm{mL})\end{array}$ & 0,125 & 0,25 & 0,375 & 0,5 & 1 & 2 & 3 & 4 & 5 \\
\cline { 2 - 11 } & & & 15 & 20 & 23 & 33 & 39 & 40 & 40 & - \\
50 & 8 & 15 & 29 & 40 & 49 & 66 & 76 & 78 & 78 & - \\
100 & 15 & 43 & 59 & 72 & 101 & 117 & 120 & 121 & - \\
150 & 25 & 43 & - \\
200 & 34 & 59 & 80 & 95 & 134 & 153 & 156 & 157 & - \\
250 & 41 & 74 & 99 & 122 & 170 & 198 & 202 & 203 & - \\
300 & 51 & 92 & 124 & 149 & 207 & 239 & 243 & 244 & - \\
350 & 57 & 102 & 135 & 165 & 230 & 269 & 274 & 275 & - \\
400 & 64 & 115 & 157 & 190 & 270 & 315 & 324 & 325 & - \\
450 & 66 & 120 & 165 & 200 & 285 & 345 & 354 & 355 & - \\
500 & 72 & 130 & 179 & 218 & 319 & 385 & 401 & 404 & 405 \\
550 & 73 & 132 & 184 & 225 & 335 & 412 & 430 & 434 & 435 \\
600 & 75 & 138 & 191 & 235 & 355 & 445 & 470 & 479 & 480 \\
\hline
\end{tabular}

Tabla III

Volumen de metano acumulado $(\mathrm{mL})$ en función del tiempo (días)

Reactor Bentonita

\begin{tabular}{crrrrrrrrrr}
\hline $\begin{array}{c}\text { Volumen de agua } \\
\text { residual } \\
\text { (mL) }\end{array}$ & 0,125 & 0,25 & 0,375 & 0,5 & 1 & 2 & 3 & 4 & 5 \\
\cline { 2 - 11 } & & & & & & & & & & \\
50 & 5 & 10 & 14 & 17 & 26 & 34 & 36 & 37 & - \\
100 & 11 & 20 & 28 & 35 & 53 & 67 & 71 & 73 & - \\
150 & 16 & 30 & 42 & 52 & 81 & 105 & 114 & 115 & - \\
200 & 22 & 38 & 56 & 69 & 105 & 133 & 144 & 145 & - \\
250 & 28 & 52 & 71 & 88 & 135 & 175 & 189 & 190 & - \\
300 & 33 & 61 & 84 & 104 & 160 & 204 & 221 & 222 & - \\
350 & 36 & 68 & 95 & 117 & 180 & 234 & 253 & 255 & - \\
400 & 41 & 77 & 108 & 135 & 210 & 275 & 295 & 304 & 305 \\
450 & 43 & 78 & 109 & 138 & 219 & 293 & 319 & 328 & 330 \\
500 & 45 & 83 & 116 & 147 & 240 & 335 & 370 & 382 & 385 \\
550 & 45 & 82 & 117 & 149 & 241 & 341 & 389 & 407 & 410 \\
600 & 44 & 81 & 114 & 145 & 245 & 357 & 411 & 446 & 450 \\
\hline & & & & & & & & &
\end{tabular}

\section{DISCUSION}

Variación del volumen de metano con el tiempo

En las figuras 1 y 2 se representan los valores del volumen de metano acumulado $(\mathrm{mL})$ en función del tiempo (días), para algunos de los volúmenes de alimentación utilizados en los digestores con soporte Sepiolita y Bentonita respectivamente. Los resultados anteriores ponen de manifiesto:

a) El volumen de metano producido crece con el volumen de residuo añadido.

b) A igualdad de tiempo, el volumen de metano producido es diferente en los dos sistemas estudiados, siendo la Sepiolita el soporte más eficaz.
Tabla IV

Valores de la $\mathrm{DQO}_{\text {inicial }}\left(\mathrm{gO}_{2} / \mathrm{L}\right)$ y del coeficiente de velocidad, $K_{0}\left(\right.$ dias $\left.^{-1}\right)$, con sus límites de confianza para cada reactor y experimento realizado

\begin{tabular}{|c|c|c|c|c|}
\hline $\begin{array}{l}\text { Volumen } \\
\text { de agua } \\
\text { residual } \\
\text { (mL) }\end{array}$ & $\begin{array}{r}\text { SEP } \\
\mathrm{DQO}_{\text {inic. }} \\
\left(\mathrm{gO}_{2} / \mathrm{L}\right)\end{array}$ & $\begin{array}{l}\text { OLITA } \\
\mathrm{K}_{\circ}\left(\text { días }^{-1}\right)\end{array}$ & $\begin{array}{c}\mathrm{BEN} \\
\mathrm{DQO}_{\text {inic. }} \\
\left(\mathrm{gO}_{2} / \mathrm{L}\right)\end{array}$ & $K_{\circ}\left(\right.$ días $\left.^{-1}\right)$ \\
\hline 50 & 0,22 & $1,79 \pm 0,05$ & 0,24 & $1,23 \pm 0,03$ \\
\hline 100 & 0,34 & $1,89 \pm 0,04$ & 0,36 & $1,29 \pm 0,03$ \\
\hline 150 & 0,46 & $1,80 \pm 0,03$ & 0,48 & $1,18 \pm 0,02$ \\
\hline 200 & 0,58 & $1,89 \pm 0,03$ & 0,60 & $1,26 \pm 0,04$ \\
\hline 250 & 0,70 & $1,81 \pm 0,02$ & 0,72 & $1,23 \pm 0,03$ \\
\hline 300 & 0,82 & $1,88 \pm 0,01$ & 0,83 & $1,25 \pm 0,03$ \\
\hline 350 & 0,94 & $1,82 \pm 0,02$ & 0,95 & $1,21 \pm 0,02$ \\
\hline 400 & 1,06 & $1,75 \pm 0,01$ & 1,07 & $1,16 \pm 0,01$ \\
\hline 450 & 1,18 & $1,64 \pm 0,02$ & 1,19 & $1,07 \pm 0,01$ \\
\hline 500 & 1,30 & $1,55 \pm 0,01$ & 1,31 & $0,95 \pm 0,02$ \\
\hline 550 & 1,42 & $1,45 \pm 0,01$ & 1,43 & $0,87 \pm 0,02$ \\
\hline 600 & 1,54 & $1,35 \pm 0,02$ & 1,55 & $0,75 \pm 0,03$ \\
\hline
\end{tabular}

\section{Sepiolita}
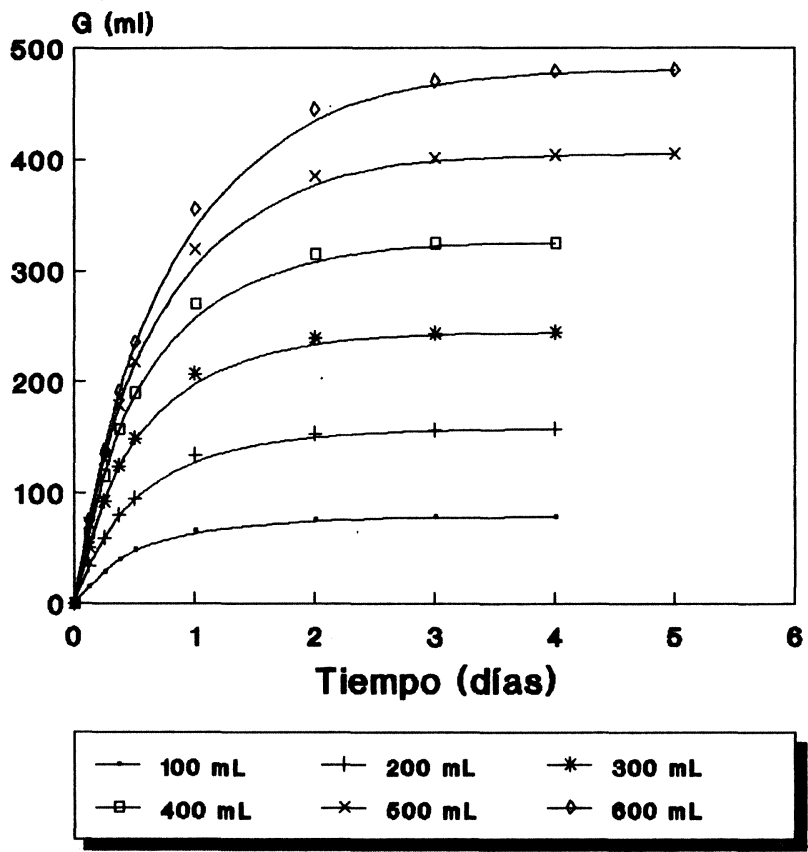

Figura 1

Volumen de metano acumulado, $\mathrm{G}(\mathrm{mL})$, en función del tiempo (dias), para algunas cargas volumétricas en el reactor con soporte Sepiolita.

A partir de los valores puntuales del volumen de metano acumulado en función del tiempo y admitiendo que, globalmente, el proceso de fermentación anaerobia sigue una cinética de primer orden (Grujer, 1983; Winkler, 


\section{Bentonita}
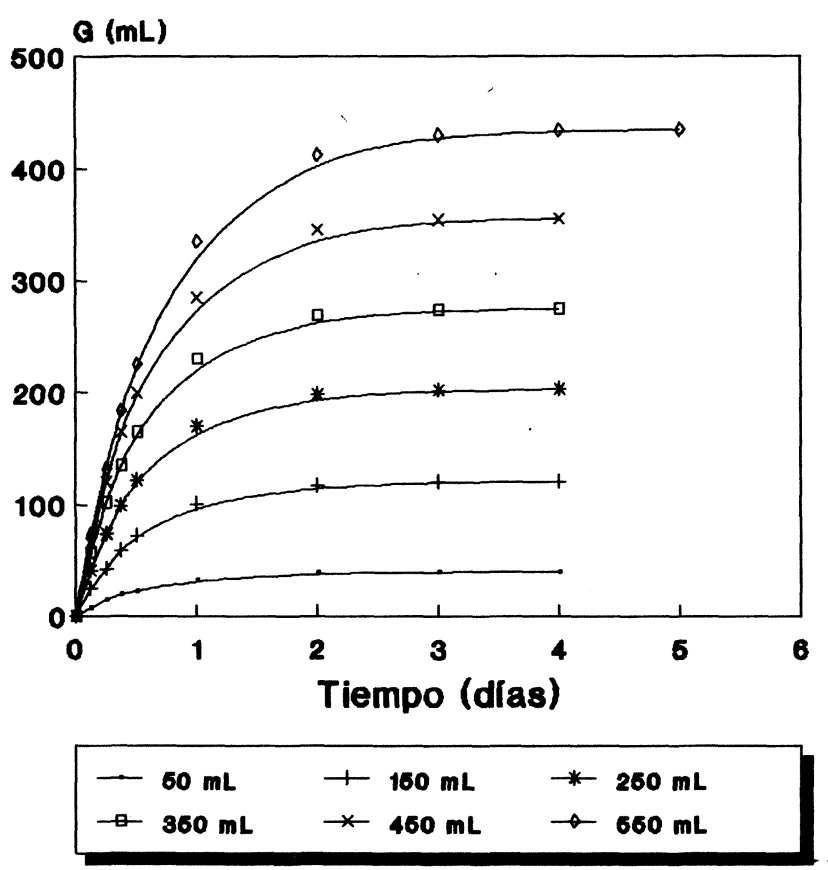

Figura 2

Volumen de metano acumulado, $G(\mathrm{~mL})$, en función del tiempo (días) para algunas cargas volumétricas en el reactor con soporte Bentonita.

1986), los coeficientes de velocidad para cada experimento realizado se obtienen mediante la ecuación de Roediger (Edeline, 1980):

$$
G=G_{m} \cdot\left[1-\exp \left(-K_{0} \cdot t\right)\right]
$$

Siendo:

$\mathrm{G}=$ volumen de metano acumulado en función del tiempo $(\mathrm{mL})$.

$\mathrm{G}_{\mathrm{m}}=$ volumen máximo de metano acumulado $(\mathrm{mL})$, considerando un tiempo de digestión infinito.

$\mathrm{K}_{\mathrm{o}}=$ coeficiente de velocidad (días $\left.{ }^{-1}\right)$.

$\mathrm{t}=$ tiempo de digestión (días).

Como se confirma en las figuras 1 y 2 , las curvas obtenidas coinciden con la que predice la ecuación anterior, decreciendo las pendientes de las mismas al aumentar el tiempo. Por consiguiente, cualitativamente, los datos experimentales responden al modelo formulado. El cálculo numérico de los parámetros $G_{m}$ y $K_{0}$ se efectúa utilizando un ajuste de regresión no lineal (Valkó and Vajda, 1989), que resulta ser estadisticamente significativo. En consecuencia, en la tabla IV se recogen los valores del coeficiente de velocidad y sus límites de confianza, para cada experimento realizado en los dos sistemas ensayados. A la vista de los resultados, se pone de manifiesto que la constante cinética, prácticamente, no es influenciada por la concentración de sustrato hasta valo- res de $D Q O_{\text {inicial }}$ de $1,0 \mathrm{~g} / \mathrm{L}$, ya que los valores de la misma están distribuidos, hasta ese límite, alrededor de la media de forma alternante. Para este tramo, los valores medios encontrados en el intervalo de concentraciones de 0 a $1 \mathrm{~g} / \mathrm{L}$, son diferentes dependiendo del soporte utilizado. Son, con un intervalo de confianza del $95 \%$, los siguientes:

\section{Sepiolita : $1,83 \pm 0,12$ \\ Bentonita: 1,23 $\pm 0,09$}

Por tanto, la constante cinética en el digestor con Sepiolita es significativamente mayor que en el de Bentonita, $(P<0,05)$.

Para densidades de carga superiores a $1,0 \mathrm{~g}$ de $\mathrm{DQO} / \mathrm{L}$, correspondientes a volúmenes mayores de 400 $\mathrm{mL}$ de agua residual, existe una disminución de la constante de velocidad, $\mathrm{K}_{0}$, al aumentar la concentración del sustrato, esto es, se produce una inhibición del proceso.

Los resultados anteriores no pueden imputarse a diferencias en la concentración de biomasa en los dos digestores, ya que ésta es idéntica en ambos (10,5 g sólidos en suspensión volátiles (SSV)/L). Sin embargo, las características y composición química del soporte pueden jugar un papel fundamental en el proceso de digestión anaerobia. Así, según Pérez Rodríguez et al., (1989), la adhesión bacteriana sobre un soporte arcilloso se puede favorecer al producirse una cesión o lenta liberación de determinados nutrientes catiónicos del soporte a los microorganismos adheridos; en concreto, el catión magnesio tiene una influencia muy favorable sobre los procesos de digestión anaerobia o biometanización (Huysman et al., 1983). Este hecho experimental puede explicar que las constantes cinéticas del proceso de depuración anaerobia de este agua residual sean más elevadas en el digestor con soporte Sepiolita, cuyo contenido en $\mathrm{MgO}$ es más alto $(23,9 \%)$ que el existente en la Bentonita $(4,6 \%)$ (Borja et al., 1990).

\section{Biodegradabilidad}

A partir de los valores de la DQO al inicio de cada uno de los experimentos realizados en los dos digestores utilizados (tabla IV), y teniendo en cuenta que la DQO de los efluentes correspondientes a cada digestor, se mantiene prácticamente constante en los valores de $0,10 \mathrm{~g} / \mathrm{L}$ (Sepiolita) y 0,12 g/L (Bentonita), se obtienen los porcentajes de sustrato biodegradado cuyos valores son superiores al $95 \%$ en ambos digestores $(95,5$ y $96 \%$, respectivamente). Este hecho pone de manifiesto la bondad de la digestión anaerobia como procedimiento para la depuración de este residuo.

Por otra parte, el $\mathrm{pH}$ de los efluentes obtenidos se mantiene prácticamente invariable en los dos digestores, presentando un valor medio de 7,2. La acidez volátil muestra una escasa variación al ir aumentando el volumen de agua residual añadida a cada digestor, presentando unos valores medios de 75 y 125 ppm de ácido acético en los soportes Sepiolita y Bentonita, respectivamente. La constancia de este parámetro y su pequeño 
valor da idea del buen desarrollo del proceso de biometanización de este agua residual.

\section{Coeficientes de rendimiento}

Dado que para cada experimento realizado se conoce el volumen de metano producido, $\mathrm{G}$, así como la DQO inicial y final, es posible calcular el coeficiente de rendimiento, $Y_{p / s}$, para cada uno de los sistemas estudiados. A partir de un ajuste lineal de las parejas de valores (G, DQO consumida) mediante el método de mínimos cuadrados, se obtienen a través de la pendiente los siguientes valores de este coeficiente de rendimiento con sus límites de confianza, considerando un intervalo de probabilidad del $95 \%$ :

\section{Sepiolita : $333 \pm 5 \mathrm{~mL} \mathrm{CH}_{4} / \mathrm{g}$ DQO \\ Bentonita: $316 \pm 6 \mathrm{~mL} \mathrm{CH}_{4} / \mathrm{g}$ DQO}

Como se observa, el valor de este coeficiente es un $5 \%$ superior en el digestor con Sepiolita. Este resultado está en consonancia con la secuencia obtenida para las constantes cinéticas del proceso, lo que nuevamente pone de manifiesto la influencia del soporte sobre el desarrollo del proceso de depuración anaerobia de este agua residual.

\section{CONCLUSIONES}

- Las aguas residuales del proceso de elaboración de aceituna de mesa negras son fácilmente biodegradables vía anaerobia, independientemente del soporte utilizado para la inmovilización de los microorganismos responsables del proceso, obteniéndose porcentajes de eliminación de DQO superiores al 95\%.

- El soporte utilizado ejerce una influencia acusada sobre la constante cinética del proceso, presentando un valor significativamente mayor en la Sepiolita, pudiéndose relacionar este comportamiento con el hecho de que el contenido en cationes $\mathrm{Mg}^{2+}$ es muy superior en este soporte al existente en la Bentonita.

- Hasta valores de 1,0 g DQO/L, los coeficientes de velocidad, $\mathrm{K}_{\mathrm{o}}$, se mantienen prácticamente invariables al aumentar la concentración de sustrato. Sin embargo, para valores superiores existe un paulatino descenso de este parámetro cinético al aumentar el volumen de residuo añadido en ambos digestores, lo que indica la existencia de un proceso de inhibición.
- El soporte Sepiolita aumenta el coeficiente de rendimiento metánico respecto a la Bentonita.

\section{AGRADECIMIENTOS}

Queremos hacer constar nuestro agradecimiento al Departamento de Postgrado y Especialización del CSIC por su ayuda para la realización de estos estudios; a la CICYT por la financiación del proyecto AU-88-0151-c0201 dentro del cual se incluye parte de esta investigación; y a D. ${ }^{\text {a }}$ Carmen Sánchez por su eficaz ayuda en la parte experimental.

\section{BIBLIOGRAFIA}

American Public Health Association (1985).- "Standard Methods for the Examination of Water and Wastewater" (16th edn.).- APHA Washington, D.C

Borja. R.; Martín, A. and Fiestas, J.A. (1990).- "Estudio Cinético de la depuración anaerobia del alpechín en presencia de diversos soportes para inmovilización de los microorganismos responsables del proceso".- Grasas y Aceites 41, 347-356

Consejo Oleicola Internacional (1989).- "Balance de la producción de aceitunas de mesa".- Comité de Expertos del COI, Madrid, Noviembre

Edeline, F. (1980).- "L'epuration biologique des eaux residuaires. Théorie et technologie".- Ed. Cebdoc-Liege, Belgium

Fernández Diez, M.J.: Castro Ramos, R. Garrido Fernández, A.; Heredia Moreno, A.; Minguez Mosquera, M.I.; Rejano Navarro, L.; Castro Gómez-Millán, A.: Durán Quintana, M.C.; Garcia Garcia, P. y Sánchez Roldán, F. (1985).- "Biotecnología de la aceituna de mesa".Consejo Superior de Investigaciones Científicas. Madrid.

Fiestas, J.A. (1984).- "Directrices actuales en la depuración de aguas residuales de carácter orgánico".- Química e Industria 30, 431-435

Grujer. W. and Zehnder, J.B. (1983).- "Conversion process in anaerobic digestion".- Wat. Sci. Tech. 15, 127-167.

Huysman, P.; Van Meenen, P.; Van Assche, P. and Verstraete, W (1983).- "Factors affecting the colonization of non porous packing materials in models upflow methane reactors".- Biotechnol. Lett. 5, 643-648.

Jeris, J. (1983).-- "Industrial wastewater treatment using anaerobic fluidized bed reactors". - Wat. Sci. Tech. 15. 123-167.

Olthof. M. and Oleszkiewick, J. (1982).- "Anaerobic treatment of industrial wastewater".- Chemical Engineering 15, 121-126.

Pérez Rodriguez, J.L.: Carretero, M.I. and Maqueda, C. (1989).- "Behaviour of Sepiolite, Vermiculite and Montmorillonite as supports in anaerobic digesters".- Appl. Clay Sci. 4, 69-82

Rozzi, A. (1988) - - "Estado del arte sobre la digestión anaerobia".- Proc III Seminario de depuración anaerobia de aguas residuales. Secretariado de Publicaciones de la Universidad de Valladolid.

Valkó. P. and Vajda, S. (1989).- "Advanced scientific computing in Basic with applications in chemistry, biology and pharmacology".- Ed. Elsevier, Amsterdam, The Netherlands.

Winkler, M. (1986).- "Biological treatment of Wastewater".- Elli Horwood Ltd., Publishers. Chapter 4.

(Recibido: Febrero 1992) 